Rome, Piazza Navona, 62

Compléments de fouilles dans les caves de l'immeuble

\title{
Martine Dewailly
}

\section{OpenEdition \\ 1 Journals}

Édition électronique

URL : http://journals.openedition.org/cefr/897

DOI : $10.4000 /$ cefr.897

ISSN : 2282-5703

\section{Éditeur}

École française de Rome

Référence électronique

Martine Dewailly, «Rome, Piazza Navona, 62 », Chronique des activités archéologiques de l'École française de Rome [En ligne], Italie centrale, mis en ligne le 23 avril 2013, consulté le 07 août 2019. URL : http://journals.openedition.org/cefr/897 ; DOI : 10.4000/cefr.897

Ce document a été généré automatiquement le 7 août 2019.

(c) École française de Rome 


\title{
Rome, Piazza Navona, 62
}

\author{
Compléments de fouilles dans les caves de l'immeuble
}

\section{Martine Dewailly}

\section{NOTE DE L'ÉDITEUR}

Ces compléments de fouilles ont été réalisés avec la participation de Julie Leone, Edwige

Lovergne, Jacopo Russo et Claudio Taffetani

1 Le projet de valorisation des caves et des espaces fouillés, élaboré sous la direction de Jean-François Bernard par les architectes Mara Colletta et Barbara Buonomo, a débuté en 2011. À mesure que les travaux avançaient, plusieurs réflexions ont été soulevées sur la rationalité et les préférences de l'itinéraire à mettre en place tout en respectant le choix des vestiges archéologiques à mettre en valeur. Cela a amené quelques modifications du projet et, en conséquence, la nécessité de compléments de fouilles : ces derniers ont été réalisés dans des espaces, prévus visitables, mais que l'ajout ou la nouvelle localisation de passerelles couvriraient tandis que, dans d'autres espaces où la fouille s'est arrêtée sur des niveaux d'occupation tardo-antiques illustrés par ailleurs, on a préféré reprendre l'enquête afin d'enrichir nos connaissances sur des problématiques peu documentées jusqu'alors, telles la réalisation du chantier de construction et l'énigmatique pavement du stade de Domitien.

2 Des sondages en profondeur ont été ouverts ou repris dans les deux salles aux piliers et dans l'ambulacre médian ${ }^{1}$.

3 Parallèlement à ces opérations ponctuelles, les différents membres de l'équipe de fouilles et les collaborateurs externes préparent le manuscrit qui constituera un volume entièrement consacré à l'exploration archéologique des caves; les données stratigraphiques et l'étude de toutes les classes de matériel restituées fourniront un exemple de la genèse, selon le thème du projet ANR, d'une portion du stade à un immeuble moderne. 


\section{Les sépultures et les niveaux sous-jacents dans la salle aux piliers sud (10401)}

\section{Les sépultures}

Les deux sépultures, situées à proximité du mur ouest et orientées ouest-est, ont été démontées en janvier 2012. Cette opération a été déterminée par l'état de conservation de la sépulture en simple fosse, mise au jour en septembre 2009 et sérieusement endommagée par les remontées successives de la nappe phréatique et, en ce qui concerne la sépulture en bâtière, par le recouvrement d'une grande partie de la pièce actuelle (fig. 1).

Fig. 1 - 62, Place Navone. Salle aux piliers sud (10401) : les sépultures (10401037 et 10401026).

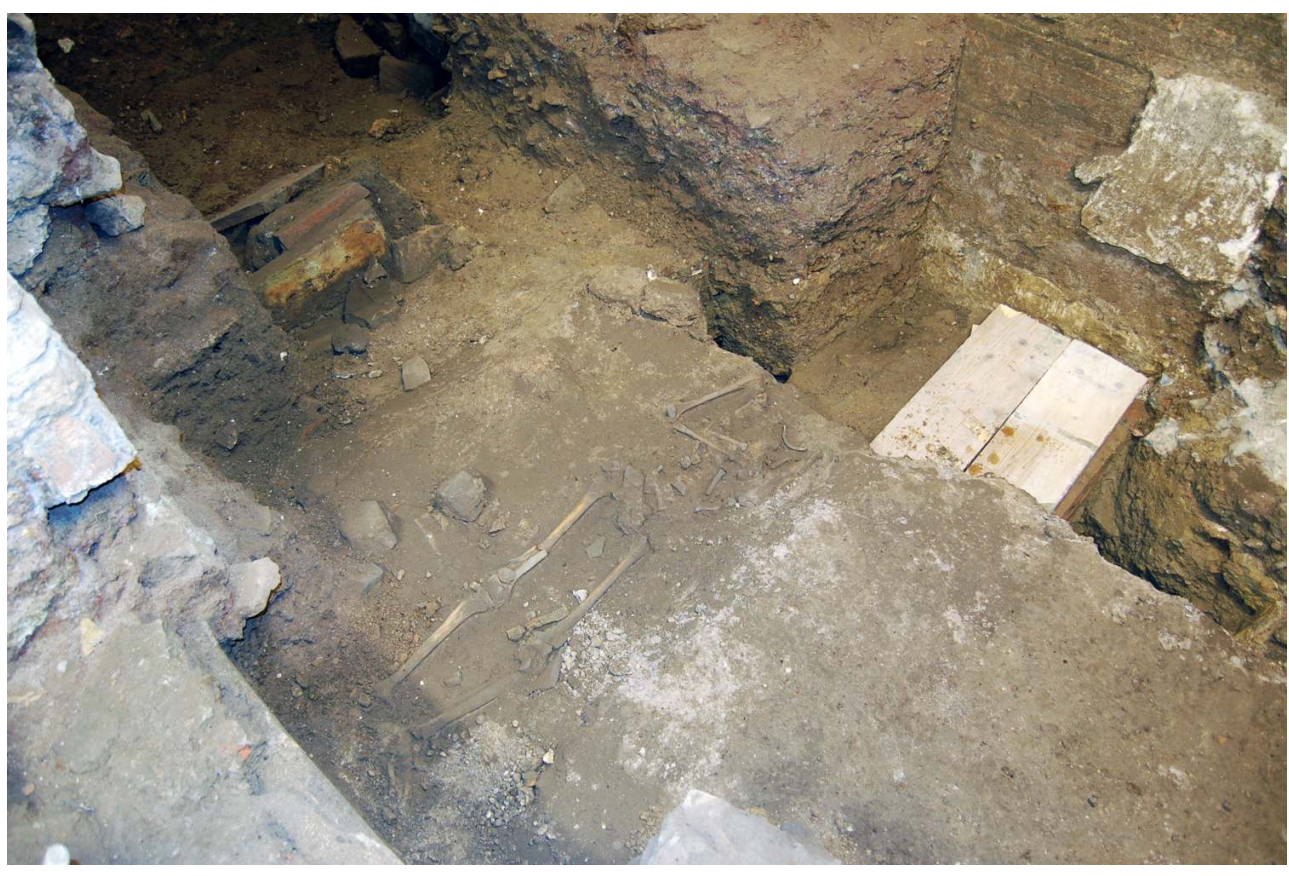

L'extrémité ouest des deux sépultures a été taillée, et les squelettes décapités, par le creusement d'une fosse-dépotoir comblée de terre mêlée à des fragments céramiques du $\mathrm{XVI}^{\mathrm{e}}$ et début du XVII ${ }^{\mathrm{e}}$ siècle; puis, celle-ci a été entamée par le creusement d'une tranchée de fondation, construite en blocage, de dimensions inférieures et d'une profondeur moindre de celles de la fosse dont les limites ont été trouvées plus à l'est et au nord. La fosse et la fondation ont été en partie arasée (cote supérieure ca. 12,80 m. alt.) lors de l'abaissement du sol des caves dans les années 1970 : la fondation était visible sur environ $0,50 \mathrm{~m}$ de hauteur. Le creusement de la fosse et son contenu correspondraient à un niveau de fréquentation contemporain du matériel recueilli et situé à une cote supérieure, sans doute proche de celle du sommet de la fondation, dont la construction est postérieure au début du XVII ${ }^{\mathrm{e}}$ siècle.

6 La sépulture 10401037 a été effectuée dans une simple fosse. La sépulture en bâtière 10401026 conservait deux tuiles posées de champ, l'une d'elles en partie affaissée, qui sont apparues englobées dans le remblai de terre rapportée, riche de céramiques tardoantiques mais mis en place au Moyen $\hat{A g e}^{2}$. Le squelette reposait sur deux autres tuiles 
dont l'une porte un timbre rectangulaire, épigraphe. Les deux sépultures étaient dépourvues de matériel relatif à l'inhumation ; le comblement de terre a restitué de rares fragments céramiques.

7 Un premier examen des restes du squelette 10401038 a été effectué par Carla Caldarini ( Servizio di Antropologia della Soprintendenza speciale per i Beni archeologici di Roma), ceux du squelette 104010027 par Giordana Amicucci (collaborateur externe de la Soprintendenza speciale per $i$ Beni archeologici di Roma).

10401038 : l'estremità occidentale della sepoltura è tagliata da un'estesa fossa di epoca rinascimentale; l'intervento antropico ha causato l'asportazione del blocco cranio-facciale, della mandibola e delle vertebre cervicali dell'inumato. Il resto dello scheletro, in cattivo stato di conservazione, si presenta in giacitura primaria ed in connessione anatomica. L'inumato è un individuo giovanile d'età alla morte compresa tra 13 e 15 anni di sesso indeterminabile. La stima dell'età alla morte ha tenuto conto del grado di saldatura diafisi-epifisi (si osserva la mancata ossificazione delle epifisi prossimali dell'omero destro e delle tibie; delle epifisi distali delle ulne e dei femori e dell'anello vertebrale) e delle misure delle lunghezze delle principali ossa lunghe. L'inumato è deposto supino con orientamento ovest-est.

10401027 : sepoltura ad inumazione in fossa terragna, provvista di copertura di tegole a cappuccina. Le estremità ovest ed est della tomba sono state entrambe tagliate, la prima da una fossa rinascimentale, che ha asportato il torace ed il cinto scapolare dello scheletro, la seconda dalla fondazione di un muro, a cui si deve l'assenza di gran parte degli arti inferiori.

Nel riempimento sono stati rinvenuti frammenti del cranio, qualche costa ed alcune ossa animali ; altri frammenti di teca cranica sono localizzati nella porzione ovest della tomba, dove si recuperano anche, dislocate, alcune vertebre toraciche e lombari.

I resti ossei sono fortemente rimaneggiati ed in cattivo stato di conservazione ; essi sono pertinenti ad un subadulto di età compresa tra i 13 ed i 19 anni, come indicato dalla mancata fusione delle epifisi alle diafisi delle ossa lunghe, e dalla non saldatura del sacro e dei coxali. La giovane età dell'individuo non consente di apprezzarne il dimorfismo sessuale, in quanto i caratteri distintivi dei due sessi non si sono ancora formati : non è pertanto possibile determinarne il sesso.

8 L'étude complète de ces deux sépultures sera publiée dans le volume dédiée à l'exploration archéologique des caves.

\section{Les niveaux sous-jacents}

9 Le creusement et le comblement de la sépulture en simple fosse et les tuiles qui formaient le fond et la couverture de la sépulture en bâtière sont scellés par le remblai rapporté. Les deux sépultures ont taillé une strate de limon sableux jaune et un niveau de circulation en mortier 10401043 (cotes 12,38 à 12,25 m alt.).

Le squelette de la sépulture 10401037 posait sur une strate charbonneuse 10401048, de limon argileux, sous-jacente au niveau de circulation (fig. 2) ; cette strate se caractérise par la présence de nombreux fragments de verre et de tesselles de pâte de verre. Une monnaie, datée de la fin du III siècle, a été trouvée en superficie. 
Fig. 2 - 62, Place Navone. Salle aux piliers sud (10401) : niveau de circulation 10401043 et, sous le squelette 10401038, la strate charbonneuse 10401048.

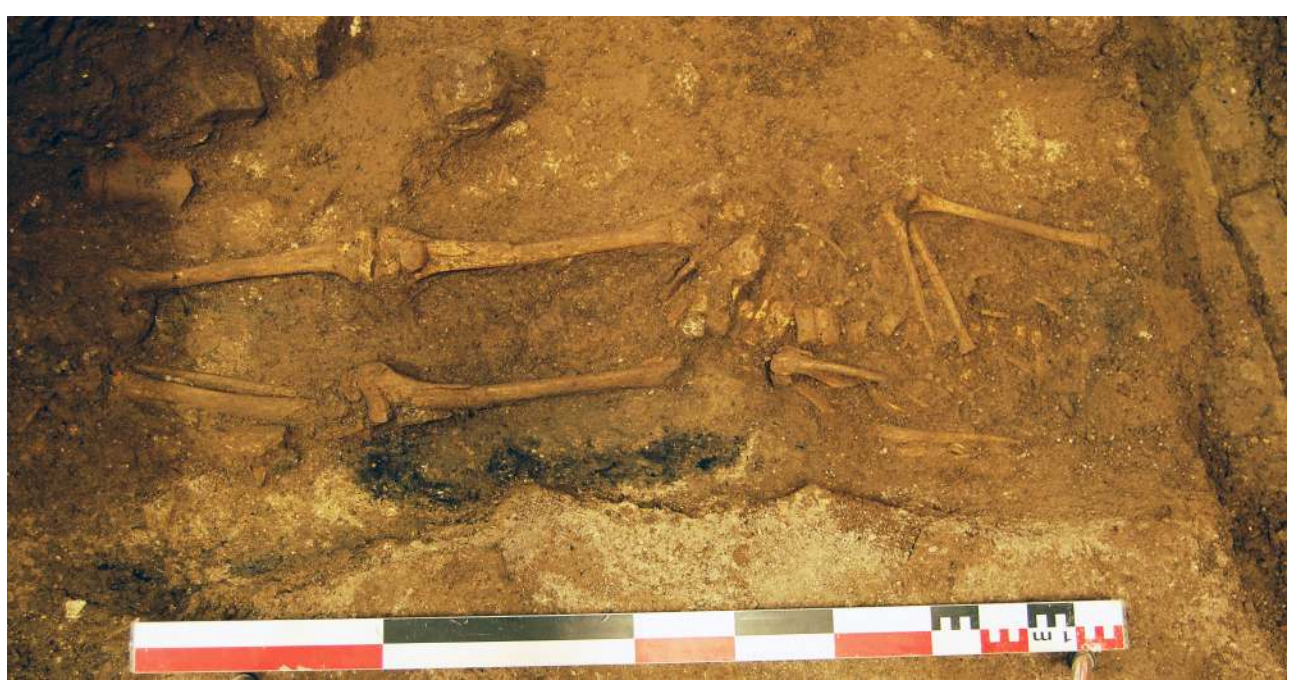

11 Les tuiles du fond de la sépulture en bâtière posaient à un niveau inférieur, sur une strate de limon sableux qui a restitué de nombreux petits fragments de bronze. Ces deux strates témoignent d'une succession d'activités artisanales. Le niveau de circulation en mortier qui les couvre est postérieur à la strate charbonneuse.

Un sondage en profondeur a été ouvert en 2009, dans la moitié nord de la salle, le long du mur ouest, au nord de la fondation radiale, puis prolongé le long du mur nord et repris en 2010. On y retrouve la strate charbonneuse précédemment décrite qui pose sur un niveau de terre compacte, mêlée à des fragments de briques, de marbres, et de mortier, dont le fond est constitué d'un lit de chaux (cotes 12,20 à 12,14 $\mathrm{m}$ alt.). Ce dernier couvre la strate de limon qui contenait des petits fragments de bronze.

13 Suit un ensemble de fines strates de limon argileux (cotes 12,15 à 12,07 $\mathrm{m}$ alt.) qui, le long du mur ouest, englobe des fragments de cipolin 10401054 (fig. 3). 
Fig. 3 - 62, Place Navone. Salle aux piliers sud (10401) : fragments de cipolin au-dessus du sol 10401058.

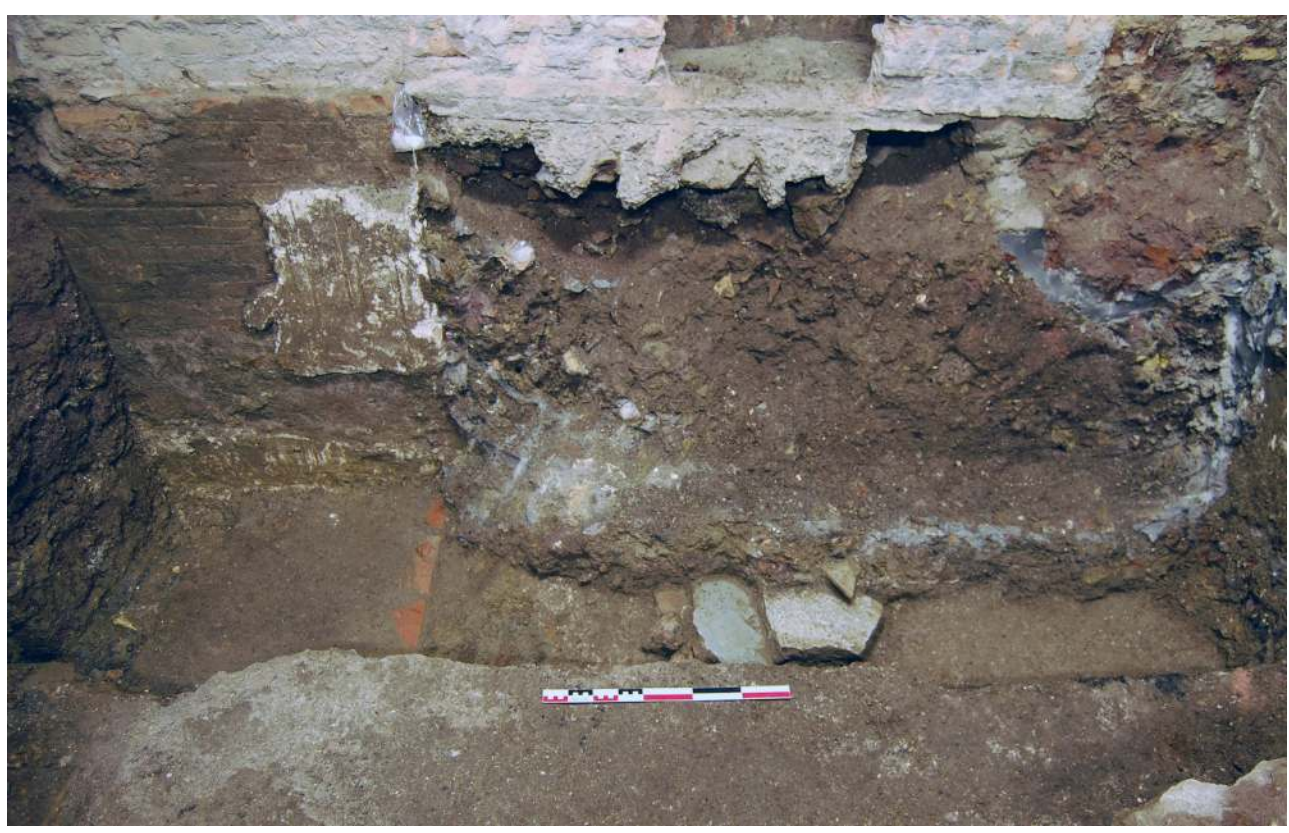

14 En même situation stratigraphique, un groupe de quatre grands fragments de marbre, dalles et fragments de gradins (?), a été trouvé en 2012 suite au démontage de la sépulture 10401037 (fig. 4); ces dalles sont situées à l'emplacement de la fondation radiale, dont elles sont séparées par une strate de préparation d'un sol.

Fig. 4 - 62, Place Navone. Salle aux piliers sud (10401) : dalles et fragments de gradins ( ?) audessus de la fondation radiale 10401046 .

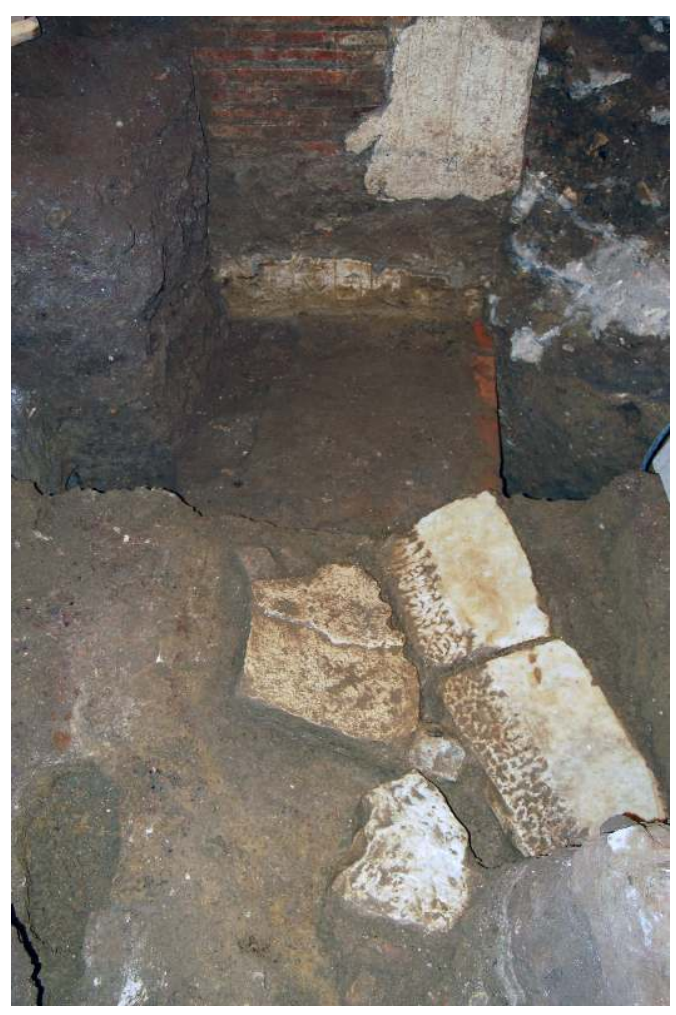



parement en briques continue au-delà du sol construit et du sommet de la fondation radiale. À ce niveau, comme dans la salle aux piliers nord, une série de fines strates de terre argileuse se superposent mais elles couvrent ici un sol très compact 10401065 (cote $11,83 \mathrm{~m}$ alt.) constitué de terre mêlée à des fragments de briques et de tuf et une préparation de sol de terre cuite compactée recouverte d'une fine strate de chaux (fig. 5). Le niveau de chantier de construction du stade est atteint à la cote 11,69 m alt.

Fig. 5 - 62, Place Navone. Salle aux piliers sud (10401) : sol 10401065 le long du mur nord.

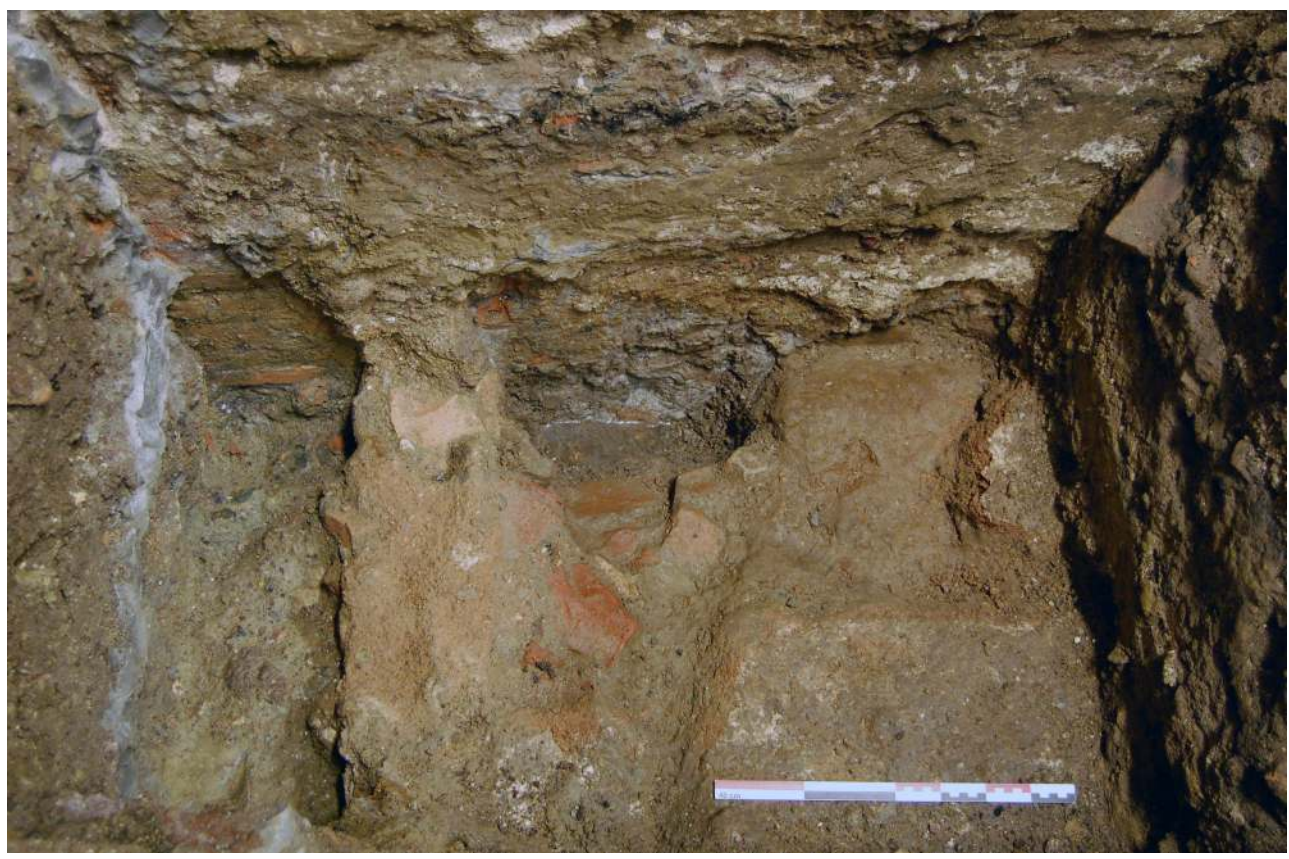

Dans la moitié sud de la salle, un sondage en profondeur, ouvert en 2010 le long du parement nord de la fondation radiale, relie le pilastre du mur ouest au mur nord de l'escalier. Le sommet de la fondation radiale situé à la cote 11,94 m. alt. était couvert d'un lit de terre cuite pilée - briques, céramiques - scellé par une strate de pouzzolane compacte, 10101033/34 (fig. 6), qui pourraient constituer une préparation à un sol du stade, non défini. Suivent, au nord de la fondation, une série de strates alternées, peu compactes - limon sableux, argile - qui correspondent à une opération de nivellement pour atteindre le niveau supérieur de la fondation. Ces strates scellent le niveau de chantier de construction du stade 10101050 situé à la cote 11,48 m. alt. (fig. 7). 
Fig. 6 - 62, Place Navone. Salle aux piliers sud (10101). Préparation de sol 10101033/34 audessus de la fondation radiale 10101029.

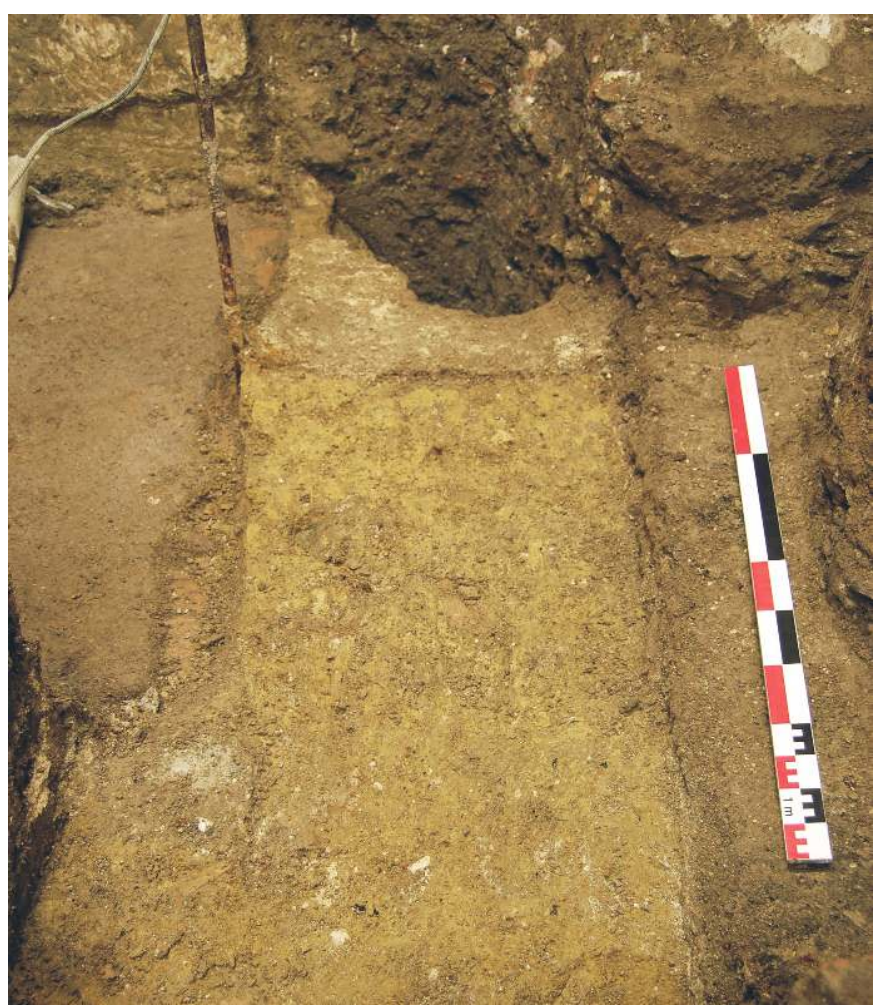

Fig. 7 - 62, Place Navone. Salle aux piliers sud (10101) : niveau de chantier 10101050.

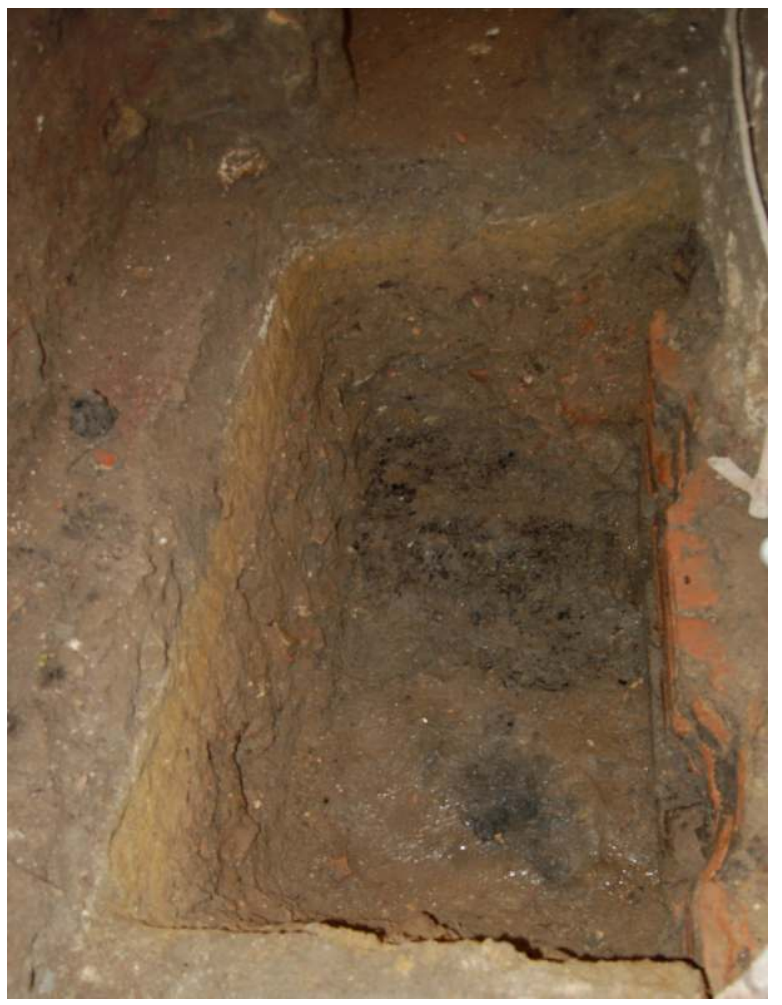

Chronique des activités archéologiques de l'École française de Rome , Italie centrale 
19 La salle aux piliers sud a donc restitué au nord un sol et sa préparation et au sud, une préparation de sol, qui couvrent les fondations radiales; on constate toutefois que leur composition n'est pas identique. Aucunes traces de pavement et de spoliation n'ont été vues.

\section{Les niveaux sous-jacents au dépôt de fragments de marbres tardo-antique (12601 et 11101)}

\section{La salle aux piliers nord}

Dans la salle aux piliers nord, le fond du dépôt de fragments de marbres se situe à $0,10 \mathrm{~m}$ au-dessus du sommet de la fondation radiale. Le sondage en profondeur, ouvert en 2010 au centre de l'espace et orienté nord-sud, a été élargi vers l'est en 2012.

En 2010, la fouille a révélé la réalisation d'une fosse immédiatement sous-jacente à la strate de fragments de marbres: la paroi est du sondage a mis en évidence que cette dernière scellait un niveau de débris de stucs qui a été taillé par le creusement de la fosse.

Deux strates ont été distinguées dans le comblement de la fosse bien que leur composition soit analogue ; le matériel céramique restitué permet de dire que cette intervention n'est pas antérieure au $\mathrm{II}^{\mathrm{e}}$ siècle ap. J.-C. Elles se superposent à une strate de remblai de terre argileuse qui couvre le niveau de chantier du stade 12601086, plan, très compact, composé de pouzzolane et de terre et apparu à 11,52 s.l.m. (fig. 8).

Fig. 8 - 62, Place Navone. Salle aux piliers nord (12601) : niveau de chantier 12601086.

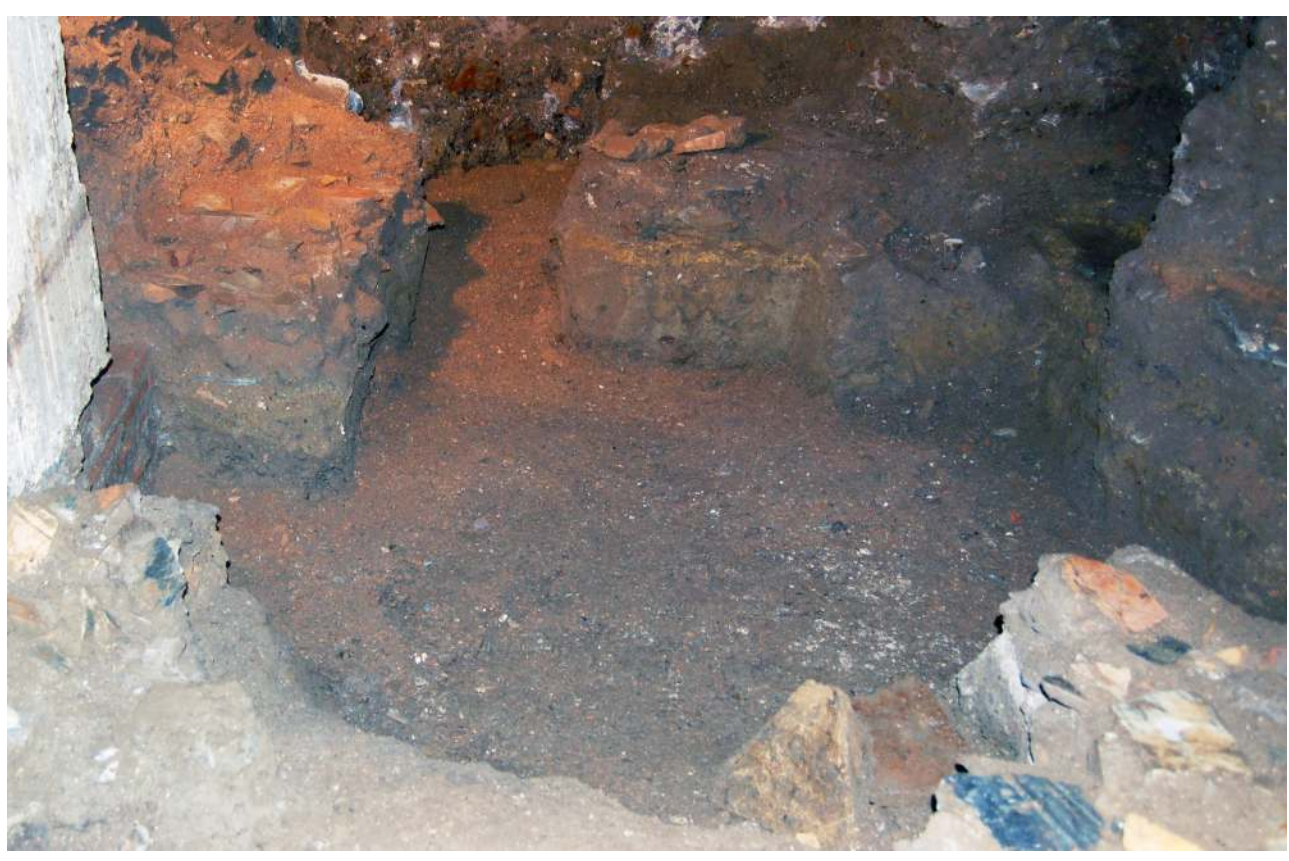

En 2012, un sondage, orienté ouest-est, a été ouvert perpendiculairement au précédent. Le niveau de débris de stucs 12601091 s'épaissit vers l'est; il couvre une première strate de remblai, éliminée plus à l'ouest par la fosse. Suit la strate inférieure de remblai de terre argileuse qui scelle le niveau de chantier (fig. 9). Le matériel provenant de ces strates de nivellement 12601098 et 12601099 est en cours d'étude. 
Fig. 9 - 62, Place Navone. Salle aux piliers nord (12601) : strate de débris de stucs 12601091 et remblai 1601098/099.

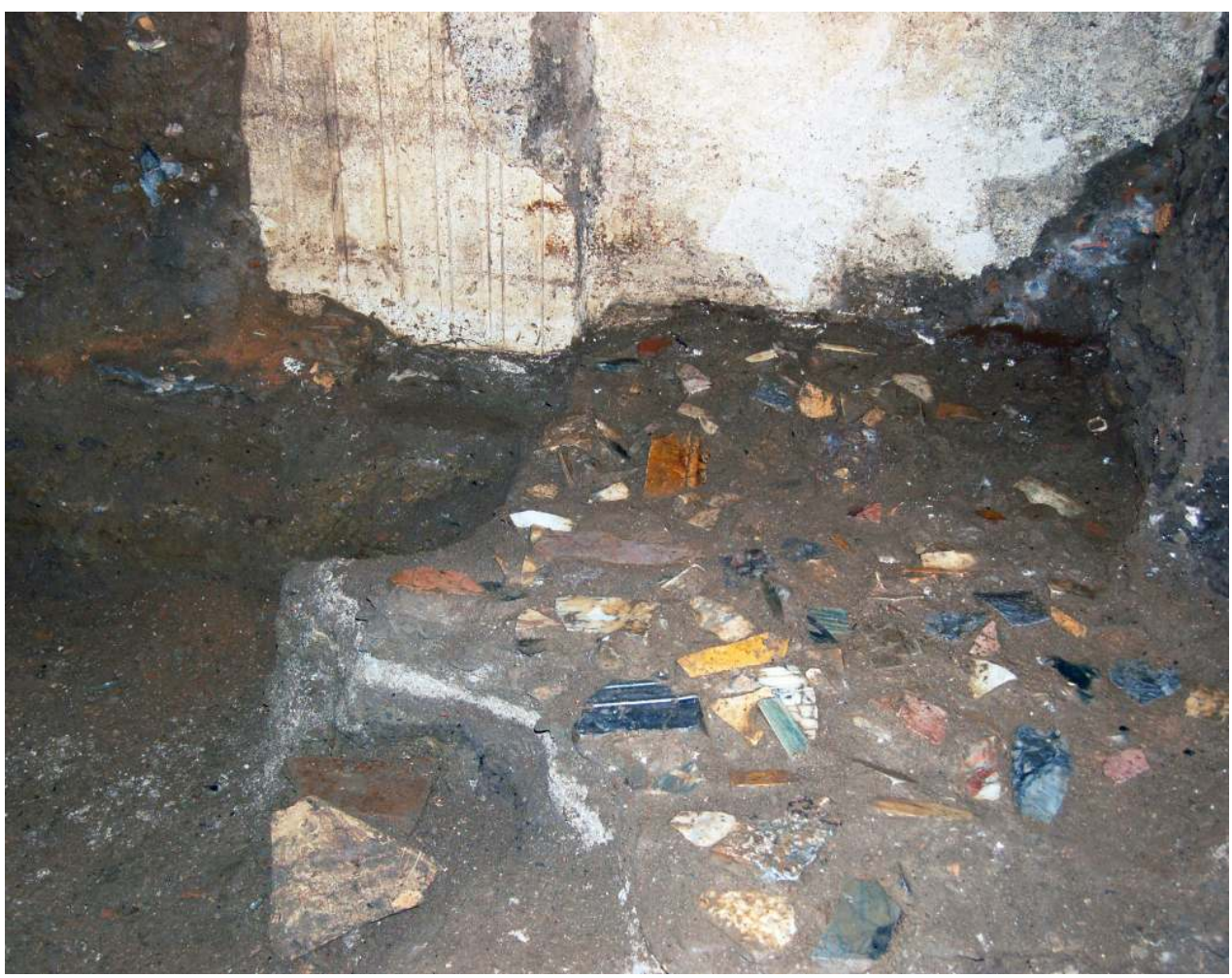

Ces deux sondages semblent permettre d'interpréter la strate de débris de stucs qui vient battre contre le sommet de la fondation radiale 12601100 et la strate de fragments de marbres qui la couvre comme un épisode d'abandon ( ?) suivi d'une phase d'occupation de l'espace ; elles occupent un niveau correspondant à celui du sol de fréquentation du stade, absent ici (fig. 10).

Fig. $10-62$, Place Navone. Salle aux piliers nord (12601) : strate de débris de stucs 12601091 et fondation 12601100 .

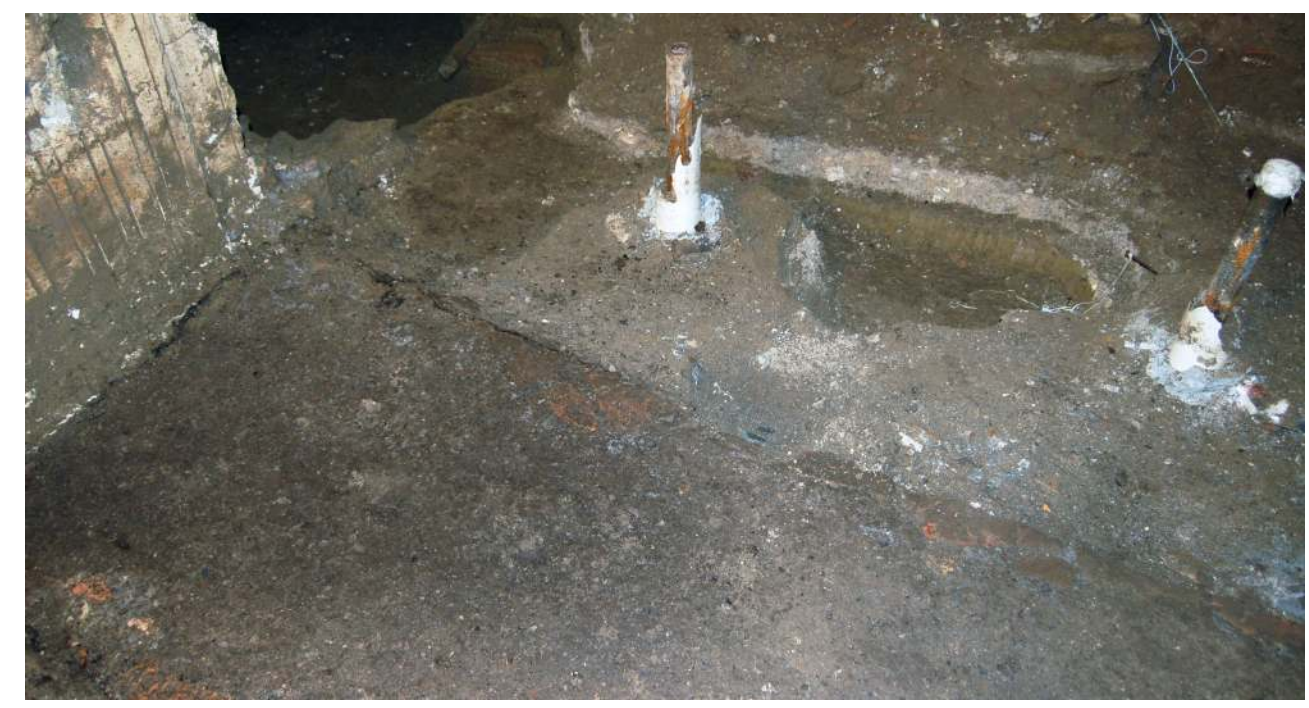



correspondant à la salle aux piliers nord, le niveau de chantier de construction du stade 11101028, apparu à 11,57/11,52 s.l.m., et les strates de remblai rapporté afin d'atteindre le sommet des fondations (cote 11,84 m. alt.) avaient été explorés sur une superficie très réduite (voir la Chronique 122.1, p. 259 et fig.41). En 2012, la fouille a été poursuivie vers le nord mais n'a pas restitué de matériel significatif (fig. 11).

Fig. 11 - 62, Place Navone. Ambulacre médian (11101) : niveau de chantier 11101028.

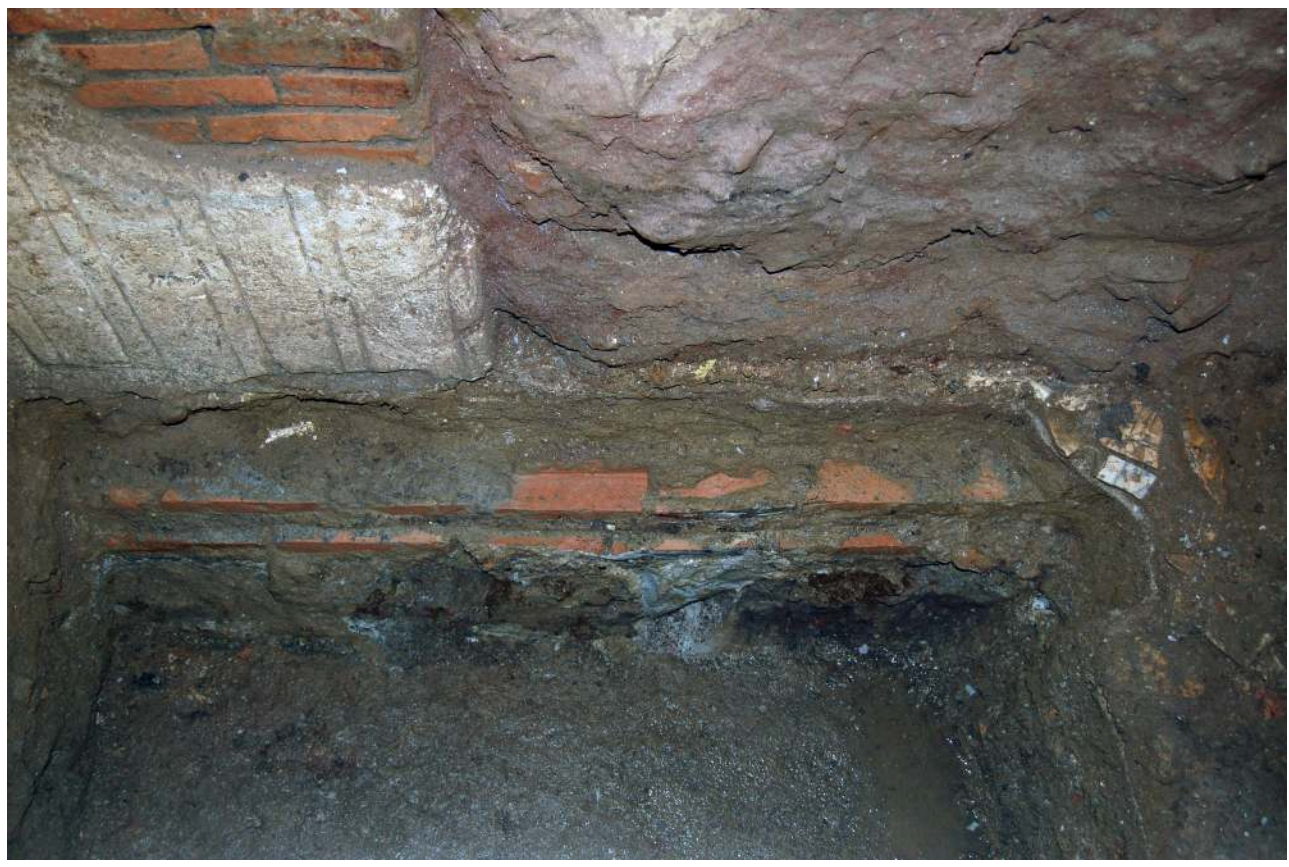

Plus au sud, dans l'axe de l'escalier antique, la fouille, arrêtée au niveau de la strate de fragments de marbres, a été poursuivie en profondeur en 2012. Un mince niveau plan, sous-jacent, a restitué plusieurs fragments d'os travaillés; il couvre une strate de terre compacte dont la surface est constituée de mortier, d'inclusions de marbre et de pouzzolane. Suit un sol de terre très compactée par la présence de très nombreux petits fragments de marbres colorés ; la strate de préparation de ce sol est constituée de grands fragments de parois d'une même amphore, posés à plat. Une épaisse strate d'argile le sépare du niveau de chantier de construction du stade, apparu à $11.46 \mathrm{~m}$. alt.

Ici, la présence d'une strate, qui témoigne d'une activité artisanale suivie d'un sol construit, rappelle la stratigraphie observée dans la moitié nord de la salle aux piliers sud.

Il est clair que ces compléments de fouilles ont été très bénéfiques car ils complètent, nuancent et/ou confortent les données obtenues lors des campagnes de fouilles réalisées entre 2006 et 2010, notamment, dans cette portion du stade, l'absence de pavement et une utilisation des espaces pour des activités artisanales dès la fin du $\mathrm{III}^{\mathrm{e}}$ ou début du IV ${ }^{\mathrm{e}}$ siècle. 


\section{NOTES}

1. Pour la localisation des espaces 12601, 10903-111 et 10401-10101, voir MEFRA 122-1, 2010, p. 257 fig. 35.

2. Cf. MEFRA 121-1, 2009, p. 297-314.

\section{INDEX}

institutions École française de Rome, Soprintendenza speciale per i Beni Archeologici di Roma Mots-clés : stade de Domitien, céramique, marbre, architecture, décor, sépulture, Moyen Âge, Navona

\section{AUTEUR}

\section{MARTINE DEWAILLY}

École française de Rome - martine.dewailly[at]efrome.it 